\title{
Comparative analysis of augmented datasets performances of age invariant face recognition models
}

\author{
Kennedy Okokpujie ${ }^{1}$, Etinosa Noma-Osaghae ${ }^{2}$, Samuel Ndueso John ${ }^{3}$, Charles Ndujiuba ${ }^{4}$, \\ Imhade Princess Okokpujie ${ }^{5}$ \\ ${ }^{1,2}$ Department of Electrical and Information Engineering, Covenant University, Ota, Nigeria \\ ${ }^{3}$ Department of Electrical and Electronic Engineering, Nigerian Defence Academy, Kaduna, Nigeria \\ ${ }^{4}$ Department of Electrical and Electronic Engineering, Air Force Institute of Technology, Kaduna, Nigeria \\ ${ }^{5}$ Department of Mechanical Engineering, Covenant University, Ota, Nigeria
}

\begin{abstract}
Article Info
ABSTRACT

Article history:

Received Aug 23, 2020

Revised Oct 12, 2020

Accepted Apr 14, 2021

\section{Keywords:}

Age invariant face recognition Convolutional neural network

Data augmentation

Fg-Net dataset

Morph dataset

The popularity of face recognition systems has increased due to their noninvasive method of image acquisition, thus boasting the widespread applications. Face ageing is one major factor that influences the performance of face recognition algorithms. In this study, the authors present a comparative study of the two most accepted and experimented face ageing datasets (FG-Net and morph II). These datasets were used to simulate age invariant face recognition (AIFR) models. Four types of noises were added to the two face ageing datasets at the preprocessing stage. The addition of noise at the preprocessing stage served as a data augmentation technique that increased the number of sample images available for deep convolutional neural network (DCNN) experimentation, improved the proposed AIFR model and the trait aging features extraction process. The proposed AIFR models are developed with the pre-trained Inception-ResNet-v2 deep convolutional neural network architecture. On testing and comparing the models, the results revealed that FG-Net is more efficient over Morph with an accuracy of $0.15 \%$, loss function of $71 \%$, mean square error (MSE) of $39 \%$ and mean absolute error (MAE) of $-0.63 \%$.
\end{abstract}

This is an open access article under the CC BY-SA license.

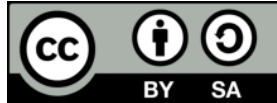

\section{Corresponding Author:}

Kennedy Okokpujie

Department of Electrical and Information Engineering

Covenant University

Km 10 Idiroko Road, Ota, Ogun State, Nigeria

Email: kennedy.okokpujie@covenantuniversity.edu.ng

\section{INTRODUCTION}

The paper aim at carrying out a comparative analysis of augmented datasets (FG-Net dataset and morph datasets). Both performances (accuracy, loss function, mean square error (MSE), and mean absolute error (MAE)) for trait-ageing invariant face recognition (AIFR) systems are compared. The significate of the study is that both datasets are used for AIFR. Data augmentation via the addition of noises to both datasets at the preprocessing phase greatly increases the accuracy and other parameters of AIFR.

- Literature review

Many comparisons exist in the literature between the performances of augmented datasets on age invariant recognition systems. The augmented dataset is usually used independently of each other to verify the invariability of designed face recognition systems. Two of the most common face image datasets used in age-invariant face recognition, FG-NET and MORPH [1] are usually at the centre of comparisons made to check the performance of age-invariant face recognition system. The goal is to have a good performance for 
all datasets used for face recognition. The results got from augmented standard datasets [2] for face images are usually based on the robustness of designed face recognition system models to variations in pose, illumination, shape, and texture. It is customary to have several ways of augmenting the datasets based on the goal of the researcher and the challenge that needs to be solved.

Comparisons between the performance of augmented datasets on age-invariant face recognition systems extend to niche applications like finding missing children who are discovered at a much later time (longer than ten years) [3]. The importance of comparisons, especially for niche applications, is emphasized in [4]. The factors that degrade the performance of face recognition systems are so numerous that it is sensible to have as many augmented datasets from as many providers as possible. The abundant evidence of the robustness of any age-invariant face recognition system is usually presented after it has passed the rigorous condition of being subject to varieties of augmented datasets [5]. The evidence is generally in the form of performance metrics like accuracy [6]. These performance metrics are used to gauge how well face recognition systems can accurately recognize face images of various subjects regardless of the source of the image, the noise added to the image and other forms of augmentation.

The region of the face used [7] to develop the age-invariant face recognition model plays a significant role in the design of age-invariant face recognition systems that are robust. The region of the face, when extracted from various datasets, could give non-identical performances on the designed face recognition model. This submission extends to other face recognition models designed to checkmate the negative effect of trait ageing. At the centre of comparisons of augmented datasets is accuracy [8], [9]. The precision with which the designed face recognition model can identify subjects' facial image after they have been designed to discriminate between real and generated images, estimate age and identify subjects. New applications of age-invariant face recognition systems like soft biometrics [10] take the comparison between augmented datasets seriously. The verification/identification process is thoroughly confirmed for as many augmented datasets as possible to verify the accuracy of the face recognition system. The algorithms used to develop age-invariant face recognition systems such as support vector machine (SVM) [11], principal component analysis (PCA) and the like, perform differently for various forms of augmented datasets. The authors in [12] tested the recognition system performance of a modelled age-invariant face recognition system after passing face images through the designed and optimized adaptive neuro-fuzzy inference system (ANFIS) classifier. The reviews made in [13] and [14] give in-depth studies of the performances of various augmented datasets on designed age-invariant face recognition system. The studies focused on the challenges of face recognition as it relates to the verification of designed face recognition systems using different augmented datasets. The studies were able to identify the challenges faced by adaptive and age-invariant face recognition systems through extensive and thorough comparisons using different augmented datasets.

\section{- Fg-Net dataset specifications and complexities}

There are 1002 images of 82 various persons with ages spanning from birth to 69 years in the FGNET database. The most common age group in the dataset is within the (<41 years) age group. Some of the pictures of subjects in the FG-NET database were digitally taken recently while others were scanned copies of the original photographs taken from personal collections of the subjects. The quality of the images in the FG-NET database depends significantly on the skill of the photographer, the condition of the photograph, the sophistication of the imaging tools used and the durability of the photographic paper found in personal collections. Thus there are variations in sharpness, illumination, resolution, background, facial expression, camera angles, and facial hair. These variations make the FG-NET database a good one for AIFR research and samples of same subject (person) ranging from ages 2 to 43 is as shown in Figure 1.

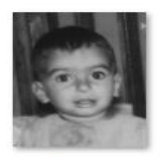

$001 \mathrm{~A} 02$

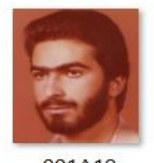

001A19

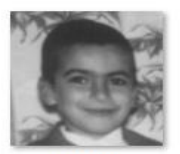

$001 \mathrm{~A} 05$

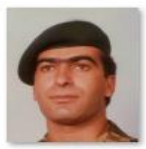

$001 \mathrm{~A} 22$
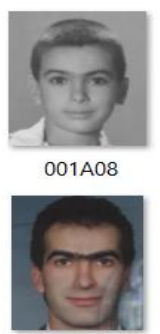

$001 \mathrm{~A} 28$

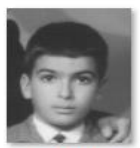

$001 \mathrm{~A} 10$

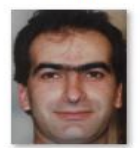

$001 \mathrm{~A} 29$

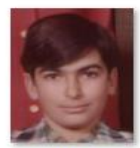

001A14

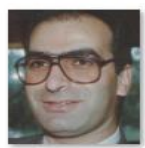

$001 \mathrm{~A} 33$

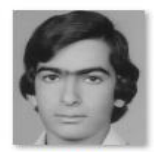

$001 \mathrm{~A} 16$

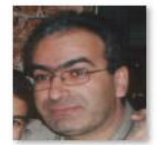

001A40

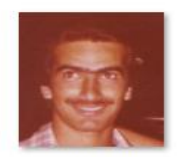

001A18

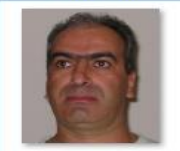

001A43b

Figure 1. Image age progression of Fg-Net dataset subject 1 at ages $1,5,8,10,14,16, \ldots \ldots .28,29,33,40,43$

\section{- Morph dataset II specifications and complexities}

A longitudinal face database, MORPH Album 2 is a well-known publicly available dataset for face recognition research. The face images in the MORPH database vary in age, sex, background. The MORPH

Comparative analysis of augmented datasets performances of age invariant face ... (Kennedy Okokpujie) 
dataset was collected in uncontrolled environments (the pictures were taken in real-world conditions) and thus has a very unique range of facial expressions. The photographs in the MORPH database were taken over a period of four years and the database is regularly updated. MORPH Album 2 contains 55,134 face images of 13,000 subjects along with metadata that shows that majority of the images were acquired in a period of four years. Example images, age progression, and statistics of MORPH Album 2, as shown in Figure 2. Figure 2(a) for white male and Figure 2(b) for african-american female.

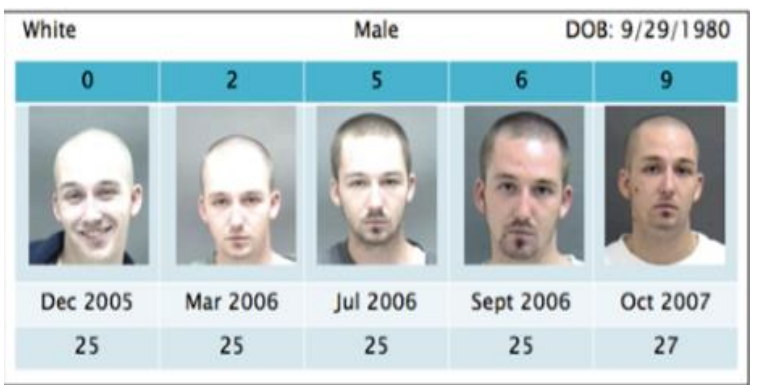

(a)

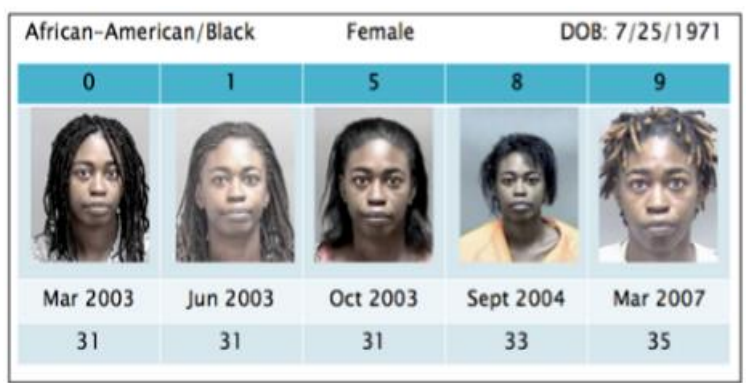

(b)

Figure 2. These figures are, (a) Image progression for white male, (b) Image progression for african-american female

- Comparative analysis of Fg-Net ageing dataset and morph dataset II

Some of the remarkable dissimilarity between the Fg-Net and the Morph datasets is that children dominate photos from Fg-Net. In contrast, most pictures from Morph are mainly from adult persons [15]. Also, the age gap between the images of the same subjects in the Fg-Net dataset is significantly wide-ranging as compare to the once in Morph dataset, which is relatively small [16], as shown in Table 1. Besides, Fg-Net contains subjects from one caucasian race, whereas Morph dataset contains the caucasoid, negroid, and mongoloid races [17]. Furthermore, the total images (samples) in Fg-Net are 1002 with 82 subjects, while that of Morph is 55,134 with 13,658 subjects [18]-[22], while details of both datasets are as shown in Table 2 and Table 3 and Table 4 depicts the Morph numbers of facial image and decade-of-life. However, the Similarity is that both datasets contain face images of the same subjects at various age gaps. The sole reason makes both ageing datasets and can be compared experimentally base on this fact [5]-[8].

Table 1. Comparison of FGNET and MORPH ageing datasets [23]

\begin{tabular}{|c|c|c|c|c|}
\hline Database & Images & Subjects & Age range & Resolution \\
\hline $\begin{array}{c}\text { FG-NET ageing } \\
\text { database }\end{array}$ & $\begin{array}{l}1,002 \text { high-resolution colour } \\
\text { or grey-scale }\end{array}$ & $\begin{array}{l}82 \text { multiple race } \\
\text { subjects }\end{array}$ & $\begin{array}{l}0 \text { to } 69 \text { years High } 294 \text { images of } \\
\text { females } 430 \text { images of males }\end{array}$ & High \\
\hline MORPH database & Album 1 1,724 face images & 515 & 46 days to 29 years & $240 \times 200$ pixels \\
\hline & Album 2 more than 20,000 & 4,000 & 16 to 77 years & - \\
\hline
\end{tabular}

Table 2. Number of sample images in each age group of FG-NET dataset [24]

\begin{tabular}{cc}
\hline Age group (in years) & Number of samples \\
\hline $0-9$ & 371 \\
$10-19$ & 339 \\
$20-29$ & 144 \\
$30-39$ & 79 \\
$40-49$ & 46 \\
$50-59$ & 15 \\
$60-69$ & 8 \\
Total & $\mathbf{1 , 0 0 2}$ \\
\hline
\end{tabular}

Table 3. Number of facial images based on gender and ethnicity from MORPH dataset [25]

\begin{tabular}{ccccccc}
\hline & African & European & Asian & Hispanic & Other & Total \\
\hline Male & 36,832 & 7,961 & 141 & 1,667 & 44 & 46,645 \\
Female & 5,757 & 2,598 & 13 & 102 & 19 & 8,489 \\
Total & 42,589 & 10,559 & 154 & 1,769 & 63 & $\mathbf{5 5 , 1 3 4}$ \\
\hline
\end{tabular}


Table 4. Morph numbers of facial image and decade-of-life [26]

\begin{tabular}{cccc}
\hline Age group (in years) & Number of samples -Male & Number of samples -Female & Total Number of Samples \\
\hline$<20$ & 6,638 & 831 & 7,469 \\
$20-29$ & 14,016 & 2,309 & 16,325 \\
$30-39$ & 12,447 & 2,910 & 15,357 \\
$40-49$ & 10,062 & 1,988 & 12,050 \\
$50+$ & 3,482 & 451 & 3,933 \\
Total & $\mathbf{4 6 , 6 4 5}$ & $\mathbf{8 , 4 8 9}$ & $\mathbf{5 5 , 1 3 4}$ \\
\hline
\end{tabular}

\section{RESEARCH METHOD}

\subsection{Pre-processing the FG-NET database for deep learning}

A mammoth amount of data is needed to train a deep neural network. The FG-NET dataset has only 10-15 face images of each subject at different ages amounting to 1002 images. The size of the FG-NET dataset is too small for deep neural network application. We preprocessed the images in the database by adding noise to it. The addition of noise to the FG-NET dataset helped increase the total amount of pictures available for deep learning application. The augmentation of the dataset was done at the preprocessing stage to allow for improved feature extraction. The following steps were followed to augment the FG-NET dataset with noise.

a. Convert all images to three channels with matrix entries for red, green an blue (RGB) for uniformity.

b. Viola-Jones face detector crops all face images and removes all background details from the face images for richer feature extraction by the proposed deep learning model.

c. Five different versions of each image is created by the addition of four types of noise namely:

- No noise (original cropped image preserved)

- Poisson noise

- Salt and pepper noise

- Speckle noise

- Gaussian noise

The number of images available for deep learning experimentation was increased from 1002 to 5010 with up to about 45-90 images per subject. The addition of noise also helped with getting the deep neural network to extract richer features from the face image for AFIR. Algorithm 1 shows the noise injection image (data augmentation) procedures.

\subsection{Pre-processing the morph database for deep learning}

A mammoth amount of data is needed to train a deep neural network. The MORPH Album 2 dataset has only 1-5 face images of each subject at different ages amounting to 13,000 images. The size of the is too small for deep neural network application. We preprocessed the images in the database by adding noise to it. The addition of noise to the MORPH Album 2 dataset helped increase the total amount of pictures available for deep learning application. The augmentation of the dataset was done at the preprocessing stage to allow for improved feature extraction. The following steps were followed to augment the MORPH Album 2 dataset with noise:

a. Convert all images to three channels with matrix entries for red, green an blue (RGB) for uniformity.

b. Viola Jones face detector crops all face images and removes all background details from the face images for richer feature extraction by the proposed deep learning model.

c. Five different versions of each image is created by the addition of four types of noise namely:

- No noise (original cropped image preserved)

- Poisson noise

- Salt and pepper noise

- Speckle noise

- Gaussian noise

The number of images available for deep learning experimentation was increased from 13,000 to 27,5000 with up to about 5-25 images per subject. The addition of noise also helped with getting the deep neural network to extract richer features from the face image for AFIR. 


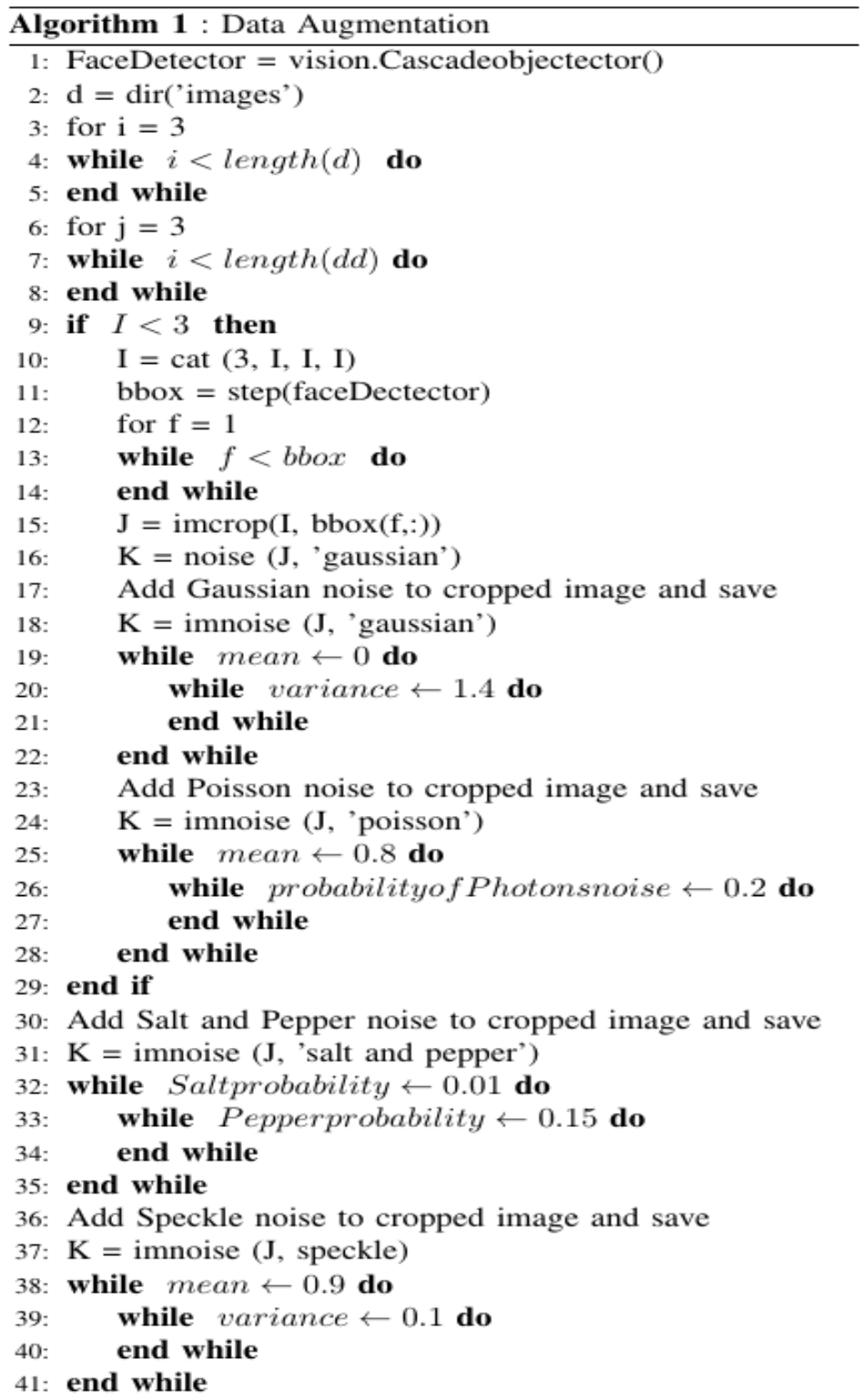

\subsection{Feature extraction and classification using convolutional neural network}

Over a million images from the ImageNet database was used to train the Inception-ResNet-v2 convolutional neural network (CNN). The images that was used to train the Inception-ResNet-v2 CNN forms part of the databased for the imagenet large-scale visual recognition challenge. Inception-ResNet-v2 has 164 layers and can classify images into 1000 object classes. The CNN accept images of size 299x299 for classification. The Inception-ResNet-v2 was used in this study to learn features for age invariant face recognition using a process called transfer learning. Transfer learning is the process of adapting a pre-trained neural network for another task for which it was not originally trained. Transfer learning was used to learn age invariant features from the FG-NET and MORPH datasets for AIFR. Figure 3 and Table 5 shows a summary of the network architecture of Inception-ResNet-v2. In order to use the Inception-ResNet-v2 network, MATLAB R2018b was installed and downloaded the installer of the deep learning toolbox model for Inception-ResNet-v2 network from [27]. Run the installer to install the Inception-ResNet-v2 network in MATLAB R2018b. 


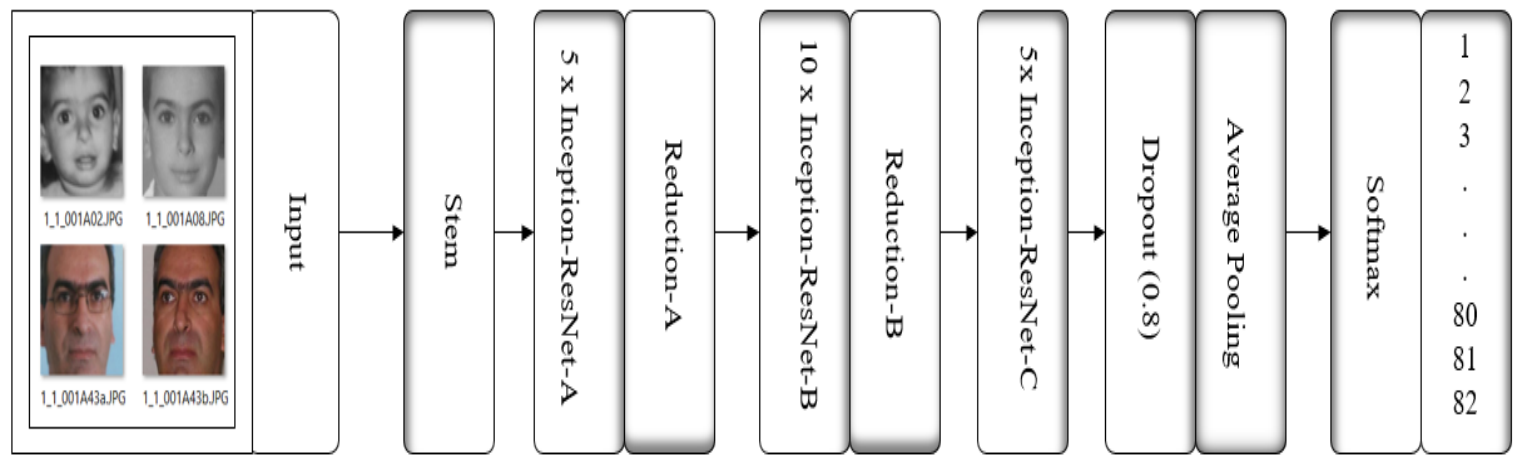

Figure 3. Block diagram of the adapted Inception-Resnet-V2 architecture network [28]

Table 5. Architecture of adapted Inception-Resnet-V2 network [29]

\begin{tabular}{|c|c|c|c|c|c|c|c|c|}
\hline Block & Type & Repeat & Depth & Filter/Stride & Output size & Branch 1 & Branch 2 & Branch 3 \\
\hline 1 & Convolution & & & $3 \times 3 / 2$ & $149 \times 149 \times 32$ & $(32)$ & & \\
\hline 1 & Convolution & & & $3 \times 3 / 1$ & $147 \times 147 \times 32$ & (32) & & \\
\hline 1 & Convolution & & & $3 \times 3 / 1$ & $147 \times 147 \times 64$ & (64) & & \\
\hline 1 & Max Pooling & & & $3 \times 3 / 2$ & $73 \times 73 \times 160$ & & & \\
\hline 1 & Convolution & & & $3 \times 3 / 2$ & $73 \times 73 \times 160$ & (96) & & \\
\hline 1 & Convolution & & & $3 \times 3 / 1$ & $71 \times 71 \times 192$ & $(64,96)$ & $(64,64,64,96)$ & \\
\hline 1 & Convolution & & & $3 \times 3 / 2$ & $35 \times 35 \times 384$ & (192) & & \\
\hline 1 & Max Pooling & & & $3 \times 3 / 2$ & $35 \times 35 \times 384$ & & & \\
\hline 2 & Inception-A & 5 & 3 & & $35 \times 35 \times 256$ & (32) & $\begin{array}{c}(32,32 / 2) \\
(32,48,64 / 2)\end{array}$ & \\
\hline 3 & Reduction-A & 1 & 3 & & $17 \times 17 \times 256$ & $(384)$ & $(256,256,384)$ & \\
\hline 4 & Inception-B & 10 & 3 & & $17 X 17 X 896$ & $\begin{array}{c}(192) \\
(256,384 / 2)\end{array}$ & $(128,160,192)$ & \\
\hline 5 & Reduction-B & 1 & 3 & & $8 \times 8 \times 1792$ & $\begin{array}{c}(256,288 / 2) \\
(256,288,320 / 2)\end{array}$ & & \\
\hline 6 & Inception-C & 5 & 3 & & $8 \times 8 \times 1792$ & (192) & $(192,224,256)$ & \\
\hline 7 & $\begin{array}{l}\text { Average } \\
\text { Pooling }\end{array}$ & & & $8 \times 8$ & 1792 & & & \\
\hline 8 & Dropout & & & Keep 0.8 & 1792 & & & \\
\hline 9 & Softmax & & & Classifier & $82 / 13000$ & & & \\
\hline
\end{tabular}

\subsection{Training the deep learning model}

The preprocessed FG-NET dataset was used to retrain the Inception-ResNet-v2 neural network for AIFR. The process was possible via Transfer learning. The transfer learning process is enumerated below:

a. The preprocessed FG-NET images are loaded into MATLAB using the image datastore object.

b. The images are then splited into a validation set (20\% images) and a train set $(80 \%)$.

c. The images in the train set are resized to $299 \times 299$ for compatipility with Inception-ResNet-v2.

d. Inception-ResNet_v2 is run by MATLAB.

e. Training preferences are specified.

f. Begin the transfer learning process using the augmented FG-NET dataset.

g. Check the validity of the transfer learning process using the validation set.

h. Estimate the network's accuracy.

\subsection{Using the trained deep learning model for face recognition in FG-NET and Morph datasets}

The retrained neural network was used for testing images from the Morph Album 2 dataset using the following process:

a. Image is read from the MORPH Album 2 dataset.

b. All images are converted into an RGB matrix

c. Viola-Jones algorithm is used to cop and detect faces.

d. All images are resized to $299 \times 299$, resize the image to $299 \times 299$.

e. The retrained Inception-ResNet-v2 neural network is loaded.

f. Load the image into the retrained neural network for classification.

g. Compare predicted class to the ground truth. 


\section{RESULTS AND DISCUSSION}

\subsection{Evaluation methodology}

MAE, MSE, Accuracy, and Loss function were used to check the performance of the proposed AIFR model [30]-[35].

\subsubsection{Accuracy}

Accuracy is derived from the true positive (TP), true negative (TN), false positive (FP), and false negative (FN) values as shown in (1). True positives are correct positive classifications. True negatives are correct negative classifications. False positives are wrong positive classifications and false negatives are wrong negative classifications.

$$
\text { Accuracy }=\frac{\mathrm{TP}+\mathrm{TN}}{\mathrm{TP}+\mathrm{TN}+\mathrm{FP}+\mathrm{TN}} * 100 \%
$$

\subsubsection{Mean squared error}

The mean squared error (MSE) is a predictor value that is always positive. A score closer to zero better. Where, $\mathrm{N}$, in this instance, is the sums of iteration, $\mathrm{f}_{-} \mathrm{i}$ is the training loss values and $\mathrm{y}_{-} \mathrm{i}$ is the testing loss values. Consequently, MSE is calculated, as presented in (2) [36], [37].

$$
M S E=\frac{1}{N} \sum_{i=1}^{N}\left(f_{i}-y_{i}\right)^{2}
$$

The MSE is the average $\left(\frac{1}{N} \sum_{i=1}^{N}\right)$ of the squares of the inaccuracies $\left(f_{i}-y_{i}\right)^{2}$.

\subsubsection{Mean absolute error}

The mean absolute error (MAE) is a measure of the disparity between two values. In this circumstance between $\mathrm{y}_{-} \mathrm{i}$ which is the values of training loss and $\mathrm{y}_{-}^{\wedge} \mathrm{i}$, which is the value of the testing loss, $n$ is the sums of iteration. Consequently, MAE is calculated, as presented in (3) [38].

$$
\operatorname{MAE}=\frac{\sum_{i=1}^{n}\left|y_{i}-\hat{y}_{i}\right|}{n}
$$

The MAE is the mean of the total errors $\left(\left|y_{i}-\hat{y}_{i}\right|\right)$.

\subsubsection{Loss function}

Categorical cross-entropy is a loss function used to calculate the variation concerning two probability disseminations. This dissimilarity is computed for respectively iteration in the training and testing dataset. The technique to calculate the likelihood variation is as shown in the (4) [39].

$$
\mathcal{L}_{\text {cross }}(y, \hat{y})=-\sum_{t=1}^{N} \cdot \sum_{i=1}^{C} y_{i}^{t} \cdot \log \left(\hat{y}_{i}^{t}\right)
$$

Where $x$ is the input value, $y$ is the true value, $\hat{y}$ is forecast value by the method, $N$ is the sum of iteration and $C$ is the sum of class labels. Wen et al. [40] recommended a loss function called centre loss in adding to using the definite cross-entropy loss. The idea is to growth the discriminative power of the completely learned features by declining the intra-class variations. The centre loss function is as shown in (5).

$$
\mathcal{L}_{\text {center }}(y, \hat{y})=\frac{1}{2} \sum_{t=1}^{N} \cdot \sum_{i=1}^{C}\left(\hat{y}_{i}^{t}-c_{y_{i}}^{t}\right)^{2}
$$

While $c_{y_{i}}$ is the $y_{i}$ th class centre of the features and $N$ is the sum of iterations. Wen et al. [40] detected that (5) seen not accomplish the expected result. Two modifications were done by Wen et al. [40] to decide this problem. First, the modification is to bring up to data the centers founded on a mini-batch as a additional for the entire dataset. For the second modification is the institution of two new variables $\alpha$ and the $\delta-$ function. $\alpha$ is used to regulate the learning rates of the centre, and the $\delta$-function is a Boolean that results in 1 if the situation is true and 0 if the situation is false. In (6) defines the updated function of the class centre.

$$
\Delta c_{j}(y, \hat{\mathrm{y}})=\frac{\sum_{t=1}^{N} \delta\left(y_{i}=j\right) \cdot\left(c_{j}-\hat{y}_{i}\right)}{1+\sum_{t=1}^{N} \delta\left(y_{i}=j\right)}
$$


The novel centre of each class is as shown in (7):

$$
c_{j}^{t+1}=c_{j}^{t}-\alpha \cdot \Delta c_{j}^{t}
$$

While $\alpha \in[0,1]$. Wen et al., [40] introduce $\lambda$ to balance the two-loss functions of the total loss function. The complete function is shown in (8).

$$
\mathcal{L}=\mathcal{L}_{\text {cross }}+\lambda \mathcal{L}_{\text {center }}
$$

In the event $\lambda$ is set to 0 , the total loss function is equal to the categorical cross-entropy function is used.

\subsection{Results and discussion}

This section deal wth the results and comparative analysis of augmented datasets (FG-Net and Morph II) performances for trait-ageing invariant face fecognition system. Figure 4 shows FG-Net and Morph datasets training, and testing accuracies results in comparative analysis. With FG-Net dataset outperforming the Morph dataset with a mean testing accuracy of $0.15 \%$. While Figure 5 shows FG-Net and Morph training and testing loss (error) results in comparative analysis. With mean FG-Net dataset output performance, the Morph dataset testing loss of $71 \%$. Table 6 shows a summary of the result performance of augmented datasets of Fg-Net and Morph dataset. All this implied that FG-Net dataset have will perform better than Morph dataset during deployment of these model in age invariant face recognition (AIFR) system

Table 6. The performance of augmented datasets (Fg-Net and Morph II)

\begin{tabular}{cccc}
\hline Variable & Fg-Net Dataset & Morph Dataset & Percentage Difference \\
\hline Accuracy (Testing) & $99.94 \%$ & $99.79 \%$ & $0.15 \%$ \\
Loss Function (Testing) & $0.0039 \%$ & $0.0067 \%$ & $71 \%$ \\
Mean Square Error (MSE) & 0.0155 & 0.0094 & $39 \%$ \\
Mean Absolute Error (MAE) & 0.0634 & 0.0638 & $-0.63 \%$ \\
\hline
\end{tabular}

Furthermore, Figure 4 emphasize in graphical form the characteristics of FG-Net and morph training and testing accuracies results in comparative analysis. While Figure 5 highlights in graphical form the attributes of FG-Net and Morph training and testing loss (error function) results in comparative analysis. Figure 6 in graphical form the characteristics of FG-Net and morph squared error results comparative analysis. Finally, Figure 7 in graphical form the attributes of FG-Net and morph absolute error results comparative analysis.

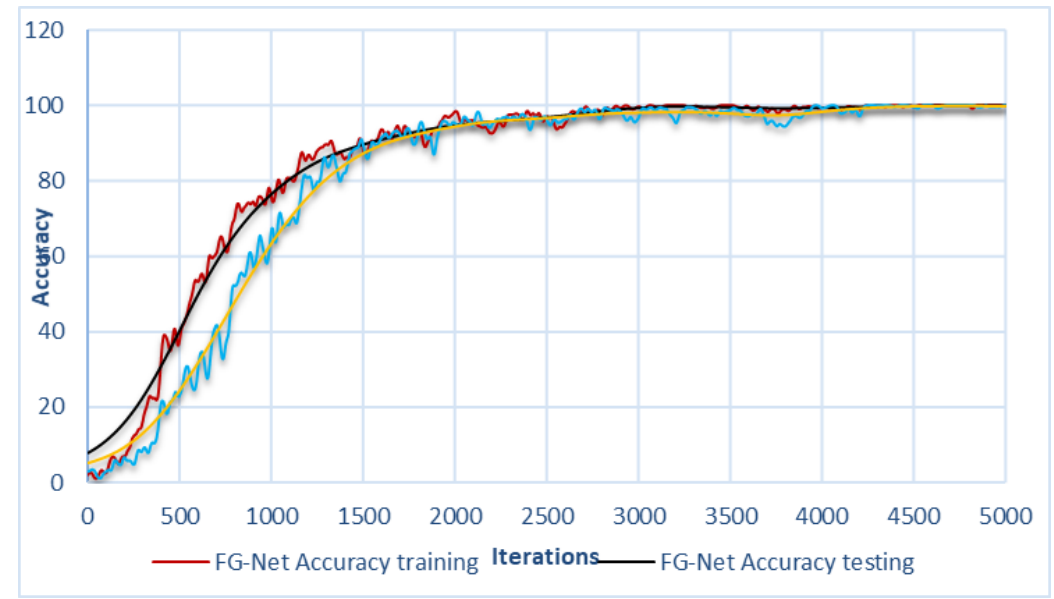

Figure 4. FG-Net and morph training and testing accuracies results comparative analysis (the result is best viewed in colour) 


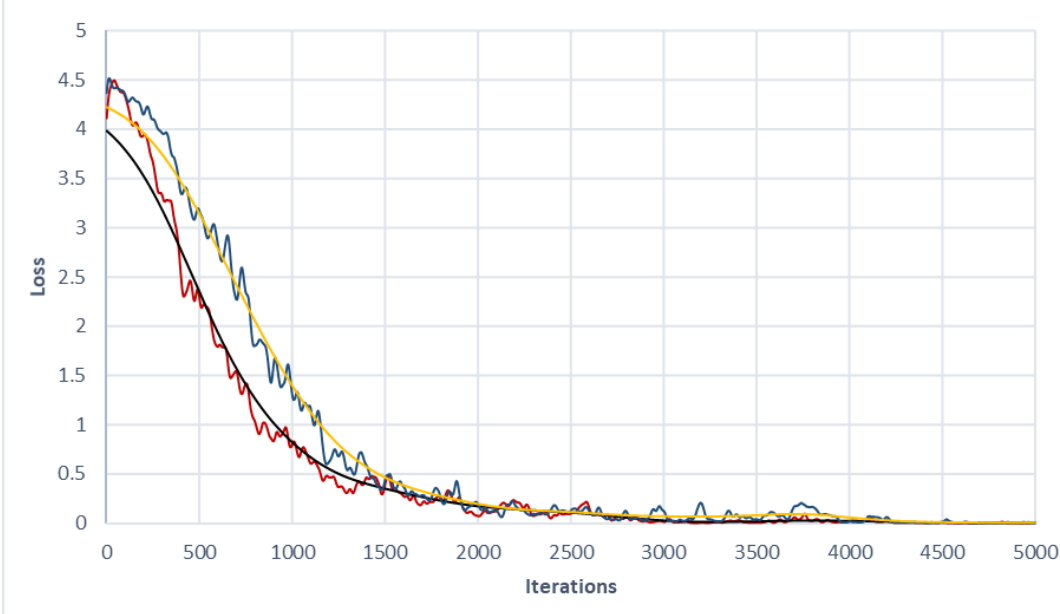

— Fg-Net Loss training — Fg-Net Loss testing —— Morph Loss training — Morph Loss testing

Figure 5. FG-Net and morph training and testing loss (error function) results in comparative analysis (the result is best viewed in colour)

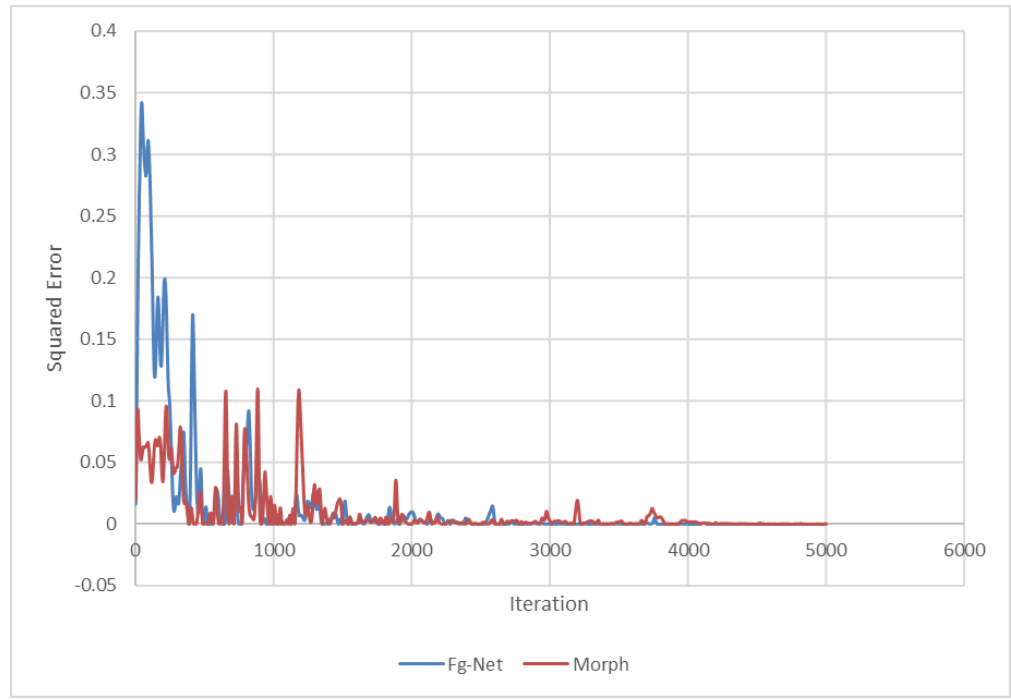

Figure 6. FG-Net and morph squared error results comparative analysis (the result is best viewed in colour)

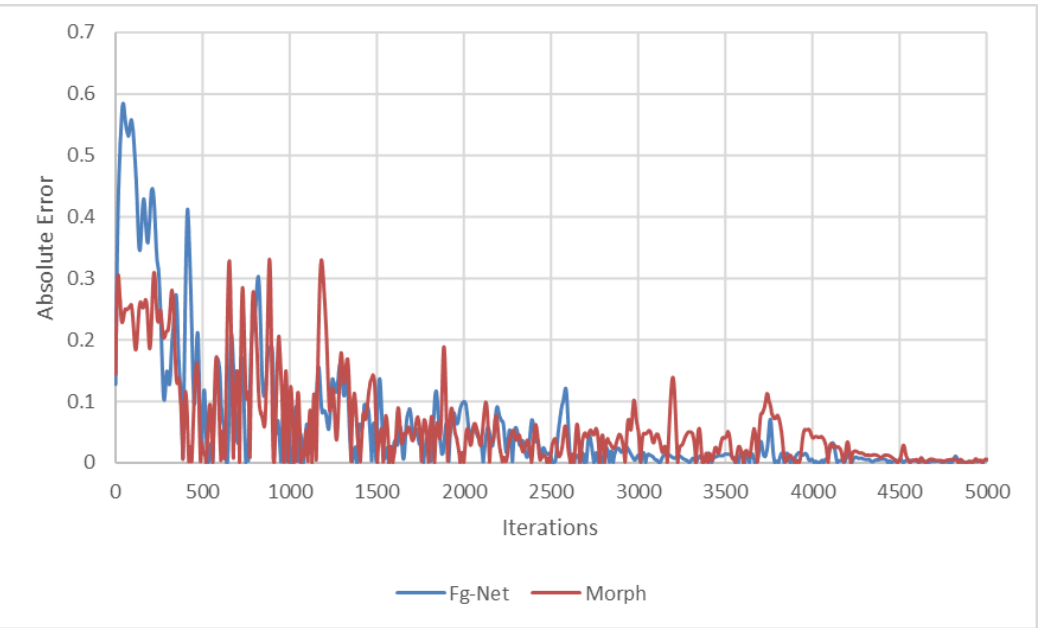

Figure 7. FG-Net and Morph absolute error results comparative analysis (the result is best viewed in colour) 


\section{CONCLUSION}

This paper compared two of the most acceptable and experimented face ageing dataset (FG-NET and Morph II). These datasets were used to simulate age invariant face recognition (AIFR) models. The obtained results show that FG-Net and Morph datasets are similar, and the little difference may be due to randomness for augmenting the dataset.

\section{ACKNOWLEDGMENTS}

This paper is sponsor by Covenant University Center of Research, Innovation, and Discovery (CUCRID) Covenant University, Ota, Ogun State, Nigeria.

\section{REFERENCES}

[1] S. Riaz, Z. Ali, U. Park, J. Choi, I. Masi, and P. Natarajan, "Age-invariant face recognition using gender-specific 3D ageing modeling," Multimed. Tools Appl., vol. 78, no. 17, pp. 25163-25183, 2019, DOI:10.1007/s11042-0197694-1.

[2] A. Yousaf, M. J. Khan, M. J. Khan, A. M. Siddiqui, and K. Khurshid, "A robust and efficient convolutional deep learning framework for age-invariant face recognition," Expert Syst., vol. 37, no. 3, 2019, doi: 10.1111/exsy.12503.

[3] D. Deb, D. Aggarwal, and A. K. Jain, "Finding Missing Children: Ageing Deep Face Features," arXiv Prepr., vol. arXiv:1911, 2019

[4] S. Anwarul and S. Dahiya, "A Comprehensive Review on Face Recognition Methods and Factors Affecting Facial Recognition Accuracy," in Proceedings of ICRIC 2019: Springer, pp. 495-514, 2020, DOI:10.1007/978-3-03029407-6_36.

[5] Y. Wu, H. Hu, and H. Li, "Age-Invariant Face Recognition Using Coupled Similarity Reference Coding," Neural Process. Lett., vol. 50, no. 1, pp. 397-411, 2019, doi:10.1007/s11063-018-9930-5.

[6] A. Caroppo, A. Leone, and P. Siciliano, "Facial Expression Recognition in Ageing Adults: A Comparative Study," Ital. Forum Ambient Assist. Living, Springer, pp. 349-359, 2018, doi: 10.1007/978-3-030-05921-7_29.

[7] K. K. Kamarajugadda and T. R. Polipalli, "Age-invariant face recognition using multiple descriptors along with modified dimensionality reduction approach," Multimed. Tools Appl., vol. 78, no. 19, pp. 27639-27661, 2019, doi: 10.1007/s11042-019-7741-y.

[8] S. Zhao, J. Li, and J. Wang, "Disentangled representation learning and residual GAN for age-invariant face verification," Pattern Recognit., vol. 100, 2019, doi: 10.1016/j.patcog.2019.107097.

[9] C. Atuegwu, S. A. Daramola, K. O. Okokpujie, and E. Noma-Osaghae. "Development of an Improved Fingerprint Feature Extraction Algorithm for Personal Verification," International Journal of Applied Engineering Research, vol. 13, no. 9, pp. 6608-6612, 2018.

[10] E. S. Jaha, "Augmenting Gabor-based Face Recognition with Global Soft Biometrics," 2019 7th International Symposium on Digital Forensics and Security (ISDFS), Barcelos, Portugal, 2019, pp. 1-5, doi: 10.1109/ISDFS.2019.8757553.

[11] P. Panchal and H. Mewada, "Robust Illumination and Pose Invariant Face Recognition System using Support Vector Machines," Int. J. Appl. Eng. Res., vol. 13, no. 16, pp. 12689-12701, 2018.

[12] S. A. Padmanabhan and N. K. Kumar, "An effective pose invariant face recognition system with the aid of ABC optimized ANFIS," Cluster Comput., vol. 22, no. 1, pp. 177-187, 2019, doi: 10.1007/s10586-018-2032-3.

[13] S. B. Ahmed, S. F. Ali, J. Ahmad, M. Adnan, and M. M. Fraz, "On the frontiers of pose invariant face recognition: a review," Artif. Intell. Rev., pp. 2571-2634, 2020, doi: 10.1007/s10462-019-09742-3.

[14] S. Singh and S. Prasad, "Techniques and Challenges of Face Recognition: A Critical Review," Procedia Comput. Sci., vol. 143, pp. 536-543, doi: 10.1016/j.procs.2018.10.427.

[15] J. Zeng, H. Ling, L. J. Latecki, S. Fitzhugh, and G. Guo, "Analysis of Facial Images across Age Progression by Humans," ISRN Mach. Vis., vol. 2012, pp. 1-7, 2012, doi: 10.5402/2012/505974.

[16] G. Panis and A. Lanitis, "An Overview of Research Activities in Facial Age Estimation Using the FG-NET Aging Database," IET Biometrics, vol. 5, no. 2, pp. 37-46, 2015, doi:10.1049/iet-bmt.2014.0053.

[17] P. Grd and M. Baca, "Creating a face database for age estimation and classification," 2016 39th Int. Conv. Inf. Commun. Technol. Electron. Microelectron. MIPRO 2016-Proc., pp. 1371-1374, 2016, doi: 10.1109/MIPRO.2016.7522353.

[18] B. Yip et al., "Preliminary Studies on a Large Face Database," 2018 IEEE International Conference on Big Data (Big Data), Seattle, WA, USA, 2018, pp. 2572-2579, doi: 10.1109/BigData.2018.8622432.

[19] M. S. Shakeel and K. M. Lam, "Deep-feature encoding-based discriminative model for age-invariant face recognition," Pattern Recognit., vol. 93, pp. 442-457, 2019, doi: 10.1016/j.patcog.2019.04.028.

[20] S. Zaghbani, N. Boujneh, and M. S. Bouhlel, "Age estimation using deep learning," Comput. Electr. Eng., vol. 68, pp. 337-347, 2018, doi: 10.1016/j.compeleceng.2018.04.012.

[21] H. El Khiyari and H. Wechsler, "Face Recognition across Time Lapse Using Convolutional Neural Networks," $J$. Inf. Secur., vol. 7, no. 3, pp. 141-151, 2016, doi: 10.4236/jis.2016.73010.

[22] H. Zhou and K. M. Lam, "Age-invariant face recognition based on identity inference from appearance age," Pattern Recognit., vol. 76, pp. 191-202, 2018, doi: 10.1016/j.patcog.2017.10.036.

[23] M. Nimbarte and K. K. Bhoyar, "Distinctive feature representations and classifiers for age invariant face recognition-a survey, " Int. J. Comput. Syst. Eng., vol. 2, no. 4, pp. 222, 2016, doi:10.1504/IJCSYSE.2016.081391. 
[24] “The FG-NET Aging Database.” [Online]. Available: http://www.fgnet.rsunit.com. [Accessed: 21-Dec-2019].

[25] "MORPH Non-Commercial Release Whitepaper." [Online]. Available: http://www.faceaginggroup.com. [Accessed: 21-Dec-2019].

[26] “Morph Dataset II." [Online]. Available: http://people.uncw.edu/vetterr/MORPH-NonCommercial-Stats.pdf. [Accessed: 24-Dec-2019].

"Inception-ResNetv2."

[Online].

Available: https://www.mathworks.com/matlabcentral/fileexchange/66607-deep-learning-toolbox-model-for-inception-resnetv2-network. [Accessed: 02-Oct-2019].

[28] K. Okokpujie, S. John, C. Ndujiuba, and E. Noma-Osaghae, "Development of an Adaptive Trait-Aging Invariant Face Recognition System Using Convolutional Neural Networks," in Information Science and Applications: Springer, pp. 411-420, 2020, doi:10.1007/978-981-15-1465-4_41.

[29] K. Okokpujie and S. Apeh, "Predictive Modeling of Trait-Aging Invariant Face Recognition System Using Machine Learning," in Information Science and Applications: Springer, pp. 431-440, 2020, DOI:10.1007/978-98115-1465-4_43.

[30] D. Wang, C. Otto, S. Member, and A. K. Jain, "Face Search at Scale : 80 Million Gallery," MSU Tech. Rep. MSUCSE-, pp. 1-14, 2015.

[31] C. Chen and A. Ross, "Matching thermal to visible face images using hidden factor analysis in a cascaded subspace learning framework," Pattern Recognit. Lett., vol. 72, pp. 25-32, 2016, doi: 10.1016/j.patrec.2015.06.021.

[32] S. Banerjee, W. J. Scheirer, K. W. Bowyer and P. J. Flynn, "Fast Face Image Synthesis With Minimal Training," 2019 IEEE Winter Conference on Applications of Computer Vision (WACV), Waikoloa, HI, USA, 2019, pp. 2126-2136, doi: 10.1109/WACV.2019.00230.

[33] B. F. Klare and A. K. Jain, "Heterogeneous Face Recognition Using Kernel Prototype Similarities," in IEEE Transactions on Pattern Analysis and Machine Intelligence, vol. 35, no. 6, pp. 1410-1422, June 2013, doi: 10.1109/TPAMI.2012.229.

[34] Y. Li, G. Wang, L. Nie, Q. Wang, and W. Tan, "Distance metric optimization driven convolutional neural network for age invariant face recognition," Pattern Recognit., vol. 75, pp. 51-62, 2018, doi: 10.1016/j.patcog.2017.10.015.

[35] F. B. Alvi and R. Pears, "A composite spatio-temporal modeling approach for age invariant face recognition," Expert Syst. Appl., vol. 72, pp. 383-394, 2017, doi: 10.1016/j.eswa.2016.10.042.

[36] Q. Zhu, P. Zhang, Z. Wang and X. Ye, "A New Loss Function for CNN Classifier Based on Predefined EvenlyDistributed Class Centroids," in IEEE Access, vol. 8, pp. 10888-10895, 2020, doi: 10.1109/ACCESS.2019.2960065.

[37] K. O. Okokpujie, E. Noma-Osaghae, O. J. Okesola, S. N. John and O. Robert, "Design and Implementation of a Student Attendance System Using Iris Biometric Recognition," 2017 International Conference on Computational Science and Computational Intelligence (CSCI), Las Vegas, NV, USA, pp. 563-567, 2017, doi: 10.1109/CSCI.2017.96.

[38] R. Angulu, J. R. Tapamo, and A. O. Adewumi, "Age estimation via face images : a survey," EURASIP Journal on Image and Video Processing, vol. 1, 2018, DOI:10.1186/s13640-018-0278-6.

[39] K. Okokpujie, E. Noma-osaghae, O. Okesola, O. Omoruyi, Osemwegie Chinonso, S. John, and I. P. Okokpujie, "Fingerprint Biometric Authentication Based Point of Sale Terminal," in Kim K., Baek N. (eds) Information Science and Applications 2018. ICISA 2018. Lecture Notes in Electrical Engineering, Springer, Singapore, vol 514, 2018, https://doi.org/10.1007/978-981-13-1056-0_24.

[40] Y. Wen, K. Zhang, Z. L. B, and Y. Qiao, "A Discriminative Feature Learning Approach for Deep Face Recognition," in European conference on computer vision. Springer, Cham, 2016, vol. 1, pp. 499-515, 2016, doi: 10.1007/978-3-319-46478-7_31.

\section{BIOGRAPHIES OF AUTHORS}

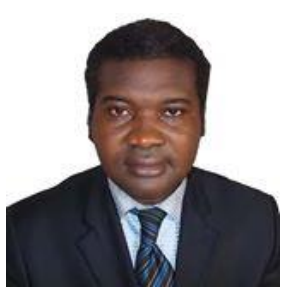

Kennedy Okokpujie holds a Bachelor of Engineering (B.Eng.) in Electrical and Electronics Engineering, Master of Science (M.Sc.) in Electrical and Electronics Engineering, Master of Business Administration (MBA), Master of Engineering (M.Eng.) in Electronics and Telecommunication Engineering and Ph.D in Information and Communication Engineering. Dr. Kennedy Okokpujie is currently a Researcher/Lecturer with the Department of Electrical and Information Engineering at Covenant University, Ota, Ogun State, Nigeria. He is a member numerous organizations including; The Nigeria Society of Engineers and the Institute of Electrical and Electronic Engineers (IEEE) and reviews for numerous high impact Journals. His research areas of interest include Biometrics, Artificial Intelligence, Communication Engineering, Digital signal Processing, Cryptography and its applications, Network security and Pravicy. 

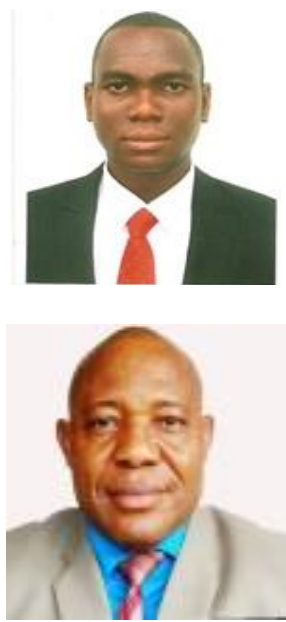
has a master's degree in Electronic and Telecommunication Engineering. Information Theory, Signal Processing, Biometrics, E-learning, E-health and Internet of Things are his research areas of interest.

Samuel Ndueso John is a Professor of Computer Systems and Network Engineering. He has his higher education at Donetsk National Technical University, Ukraine where he successfully defended and obtained B.Sc, M.SC, MPhil and Ph.D. degrees in Computer Systems and Network Engineering, specializing in Computer Science, Computing Machines, Complex Systems, Security and Networks in 1993, 1994, 2000 and 2005, respectively. Presently, John is a Professor of Computer Systems and Network Engineering in the Department of Electrical/Electronic Engineering, Faculty of Engineering and Technology, Nigerian Defence Academy, Kaduna, Nigeria. John has acquired valuable knowledge and practical experience in the use of information technology as an enabler of industrial and national development goals. He has a vast knowledge of computing and has applied it in the pursuance of a wide range of indigenous ICT Convergence, Data Efficiency Management, Cyber Security, Cybercrime and Telemedicine solutions.

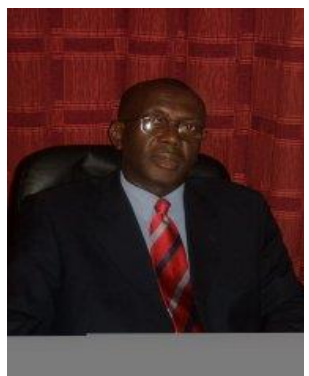

Charles U. Ndujiuba is a professor of Communication Engineering with Air Force Institute of Technology Nigerian Air Force, Kaduna state, Nigeria. Prof. C. U. Ndujiuba holds a Ph.D. in Electrical \& Electronics Engineering from the University College London (University of London); Master Specialize (Masters with Specialization) in Radio Communications from Ecole Superieure d'Electricite (SUPELEC) France; MSc in Electrical Engineering from the University of Lagos; BSc in Electronics \& Communications Engineering from the London Metropolitan University. Prof. Ndujiuba is a Chartered Electronics Engineer (CEng) and a highly skilled wireless professional. He has more than 25 years of RF, Microwave, Fixed-line (SDH and PDH), and PMR experience, with considerable international exposure. Prof. Ndujiuba has attended several conferences and published many technical papers in major professional journals. Prior to joining Covenant University Ota Nigeria in 2011, Ndujiuba was the Technical Director of Globe Trunk Ltd UK. His research interests include Monolithic Microwave Integrated Circuits (MMIC), Active Filters, Ultra-Low Noise Amplifiers, Active Devices and Circuits, UWB Transmitter, Modelling \& Simulation, Dielectric Resonator Antennas, and Detection and Collision Avoidance of Unmanned Aerial Vehicles.

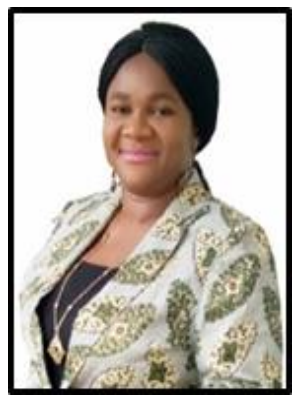

Engr. Dr. (Mrs) Okokpujie Imhade Princess is a researcher/lecturer in the Department of Mechanical Engineering Covenant University, Ota, Ogun State Nigeria. She is currently the Chief Editor to Covenant Journal of Engineering Technology (CJET). She is also a reviewer and editor of many international/local journals and conferences. Her areas of research interest are Design and Production, Advanced Manufacturing such as Machining, Tool Wear, Vibration, Nano-lubricant, Energy Systems, Mathematical Modeling, Optimization, Mechatronics and also a Multi-disciplinary Researcher. Dr. I. P. Okokpujie is an active researcher who has authored/coauthored over 106 peer-reviewed publications in reputable journals and international conferences. In 2017 to 2019, she was the technical secretary to the International Conference on Engineering for a Sustainable World (ICESW) index in Scopus and ISI data based through the IOPs publisher. She is a Registered Engineer of the Council for the Regulation of Engineering in Nigeria (COREN), a member of the Nigerian Society of Engineers (NSE), member Nigerian Institution of Mechanical Engineers and Association of Professional Women Engineers of Nigeria (APWEN). She is currently the National Technical Secretary and Vice-Chairman of APWEN Ota Chapter in Ogun State, also, the Technical Editor to Journal of Nigeria Institution of Mechanical Engineers. She is one of the top-rated researchers in her institution, and she is happily married and blessed with children. Dr. I. P. Okokpujie is very passionate about the Education of the Girl Child and also offers quality mentorship to the Young Engineers. 